\title{
The Prognostic and Clinicopathological Significance of IGF-1R in NSCLC: a Meta-Analysis
}

\author{
Jun Zhao ${ }^{a}$ Xuefeng Shi ${ }^{a}$ Tao Wang ${ }^{b}$ Chunhua Ying ${ }^{c}$ Shaojun He ${ }^{d}$ Yifei Chen ${ }^{d}$ \\ aDepartment of Respiratory Medicine, Qinghai Provincial People's Hospital, Xining, Qinghai, \\ bDepartment of Respiratory and Critical Care Medicine, Renmin Hospital of Wuhan University, Wuhan, \\ Hubei, 'Department of Ultrasound, Qinghai Provincial People's Hospital, Xining, Qinghai, dDepartment \\ of Respiratory and Critical Care Medicine, Zhongnan Hospital of Wuhan University, Wuhan, Hubei, \\ China
}

\section{Key Words}

NSCLC $・$ IGF-1R • Overall survival • Prognosis • Meta-analysis

\begin{abstract}
:
Background/Aims: Accumulating studies have reported that IGF-1R (Insulin-like growth factor-1 receptor) is aberrantly expressed in NSCLC (non-small cell lung cancer), but the role of IGF-1R in NSCLC remains controversial. The present paper assessed the precise role of IGF-1R in NSCLC. Methods: We comprehensively searched PubMed, EMBASE, and Web of Science in March 2017. Combined HRs and ORs were used to evaluate the prognostic and clinicopathological significance of IGF-1R in NSCLC respectively. Results: A total of 10 eligible studies including 8 on overall survival, and 10 on clinicopathological features were identified from the databases. The results showed that high expression of IGF-1R was associated with shorter OS (overall survival) of NSCLC patients (pooled HR 1.17,95 \% CI 1.00-1.36). In addition, we found that IGF-1R was related to smoking status ( $\mathrm{OR}=1.82,95 \% \mathrm{CI}=1.35-2.44)$ and IGF-1R tended to be highly expressed in SCC (squamous cell carcinoma) (OR=3.40 $95 \%$ CI: 1.95-5.95). Conclusions: In summary, this meta-analysis revealed that high expression of IGF-1R was associated with poor prognosis in NSCLC.

\section{Introduction}

Lung cancer is one of the major causes of cancer-related death worldwide [1, 2]. The data analysis results reported by cancer statistics 2017 estimated that 222, 500 new cases will be diagnosed with lung cancer and the death caused by lung cancer maybe 155, 870 [3]. Non-small cell lung cancer (NSCLC) is a major type of lung cancer which accounts for about $85 \%$ of all lung cancer cases [4]. Despite patients can be successfully treated by surgical 


\section{Cellular Physiology Cell Physiol Biochem 2017;43:697-704 \begin{tabular}{l|l|l} 
DOI: 10.1159/000480655 & $\begin{array}{l}\text { O 2017 The Author(s). Published by S. Karger AG, Basel } \\
\text { www.karger.com/cpb }\end{array}$
\end{tabular}}

Zhao et al.: Prognostic and Clinicopathological Significance of IGF-1R in NSCLC

resection, systemic chemotherapy and targeted drugs, the prognosis of NSCLC still remains poor $[5,6]$. Therefore, it is of great importance to discover new biomarkers to predict the prognosis and therapy targets for NSCLC.

Insulin-like growth factor-1 receptor (IGF-1R) is a transmembrane heterotetrametric protein composed of 2 extracellular $\alpha$ and $\beta$ subunits [7]. When IGF-1 and IGF-2 bind to the extracellular subunit domain of IGF-1R, the tyrosine kinase activity of IGF-1R can be activated and the activation of IGF-1R can trigger a cascade of reactions involving signal transduction pathways such as Ras, Raf and mitogen-activated protein kinase (MAPK) [8]. It is reported that the overexpression of IGF-1R can promote tumor growth, progression, invasion and metastasis [9]. A large number of studies have revealed that IGF-1R was dysregulated in a variety of cancers including breast cancer $[10,11]$, liver cancer $[12,13]$, gastric cancer [14] and lung cancer $[15,16]$, suggesting a vital role of IGF-1R in cancers.

Up to date, IGF-1R has attracted increasing attention because of its important role in promoting NSCLC progression. It has been reported by Cappuzzo et al. [17] that IGF-1R was positive in $76.4 \%$ of patients with NSCLC. A phase II study showed that agents which target at IGF-1R exhibited a promising efficacy against advanced NSCLC [18], whereas, the prognostic value of IGF-1R is still controversial. Yamamoto et al. [9] demonstrated that the overall survival (OS) in NSCLC patients with IGF-1R-high expression was significantly poorer than those in IGF-1R-low expression patients, however, Kim et al. [19] revealed that the expression level of IGF-1R in NSCLC was not related to OS. To clarify the precise prognostic and clinical value of IGF-1R in NSCLC, a meta-analysis is needed. In our study, by combing results from the available data, we sought to make an objective evaluation of the prognostic and clinical value of IGF-1R in NSCLC.

\section{Materials and Methods}

\section{Search strategy}

A literature search was performed in March 2017 in PubMed, EMBASE, and Web of Science. The key search items were "type 1 insulin-like growth factor receptor", "insulin-like growth factor 1 receptor", "IGF$1 \mathrm{R}$ " and "lung cancer", "lung tumor", "lung carcinoma", "lung neoplasm" "NSCLC" and "non-small cell lung cancer". The literature search was limited to human studies and articles written in English. Besides, we reviewed relevant reference of the retrieved articles to identify potentially eligible studies.

\section{Inclusion and exclusion criteria}

The inclusion criteria are as follows: (1) prospective or retrospective cohort studies on patients with NSCLC; (2) researches assessed the prognostic or clinical value of IGF-1R in NSCLC; (3) researches provided hazard ratios (HRs) or the information that allowed manual calculation of 95\% CI; (4) studies published in English; (5) studies detect the expression of IGF-1R using immunohistochemically method; (6) studies investigated the role of IGF-1R in NSCLC. Accordingly, studies with the following criteria were excluded: (1) studies without usable data; (2) duplicate publications; (3) reviews, letters, single case reports; (4) animal studies. When there were duplicate studies for the same population, the latest published one was included. Only in this way could we avoid overestimating the value of HR.

\section{Data extraction}

Two investigators extracted the data from eligible studies independently and the following information was collected: name of the first author, year of publication, country, number of cases, clinical stage, detecting method, smoking status, pathological type, recruitment period, HR value and follow-up duration. We summarized the characteristics of the studies into a table format.

\section{Statistical analysis}

HR and its corresponding 95\% CIs were used to assess the association between IGF-1R expression and OS in NSCLC. ORs (odds ratios) and 95\% CIs were used to evaluate the relation between IGF-1R expression and clinical features. If an article didn't provide HRs values, Engauge Digitizer version 4.1 was used to get 


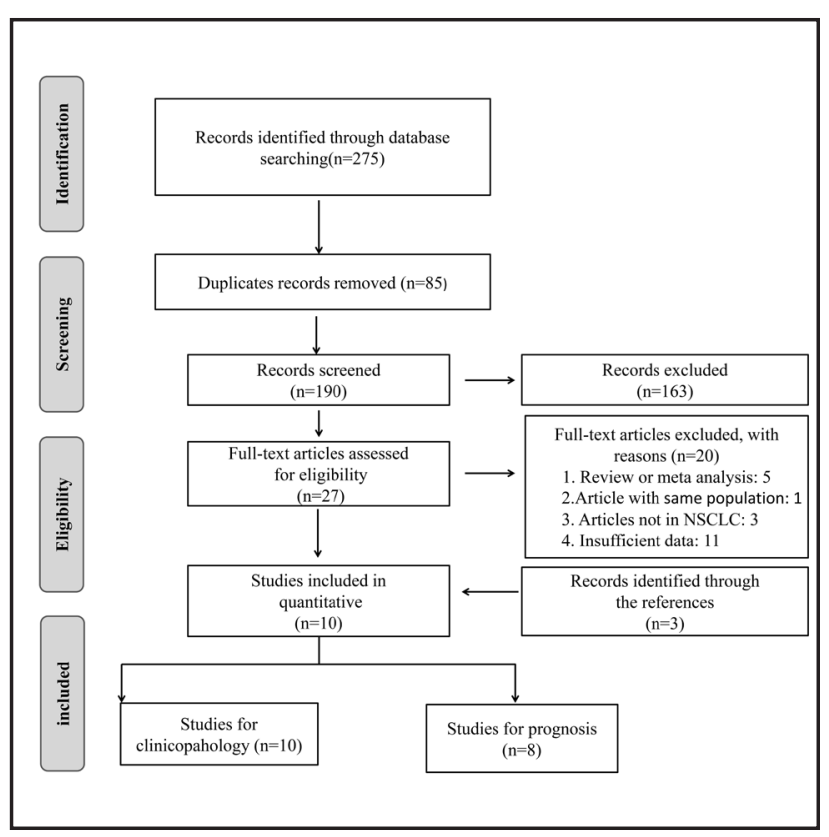

Fig. 1. Flow diagram of study search and selection process.

HRs values [20]. A fixed effect model was used when there was no obvious heterogeneity $\left(I^{2} \leq 50 \%\right)$, otherwise, a random effect model was applied [21]. Sensitivity analysis was performed to test the reliability of the total pooled results by omitting the study sequentially. Begg's test was used for assessing the publication bias. Two-sided $P<0.05$ was considered to be statistically significant. All analyses were carried out using Review Manager Version 5.3 or STATA software version 12.0 (Stata Corporation, College Station, Texas, USA).

\section{Results}

\section{Study selection and characteristics}

As shown in Fig.1, a total of 275 records were retrieved from PubMed, EMBASE and Web of Science, after we removed duplicates, 190 articles were remained to be screened. Then 163 studies that report on unrelated topics were excluded after we screened the titles and abstracts. Subsequently, the 27 remaining full-text articles were reviewed in depth and 20 studies including 5 review or meta-analysis, 1 article with same population, 3 studies evaluated IGF-1R expression that not in NSCLC, 11 with insufficient data were further excluded according to the exclusion criteria. Then 3 articles were identified through reviewing the references. Finally, a number of 10 articles were included in the current meta-analysis including 10 studies for clinicopahology and 8 studies for prognosis.

Table 1 summarizes the main characteristics of the included studies. A total number of 1958 patients with the highest number of 379 and the smallest number of

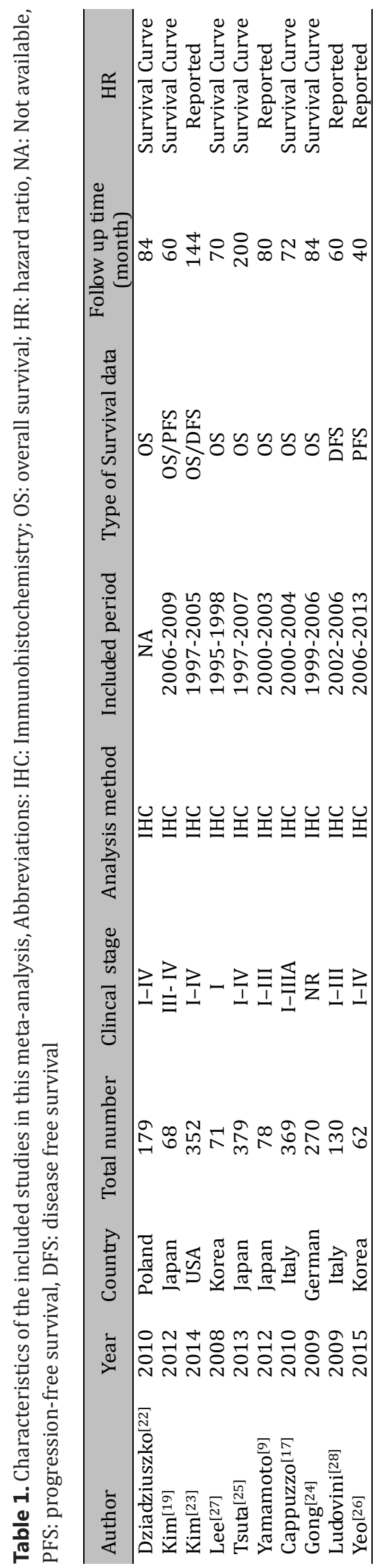




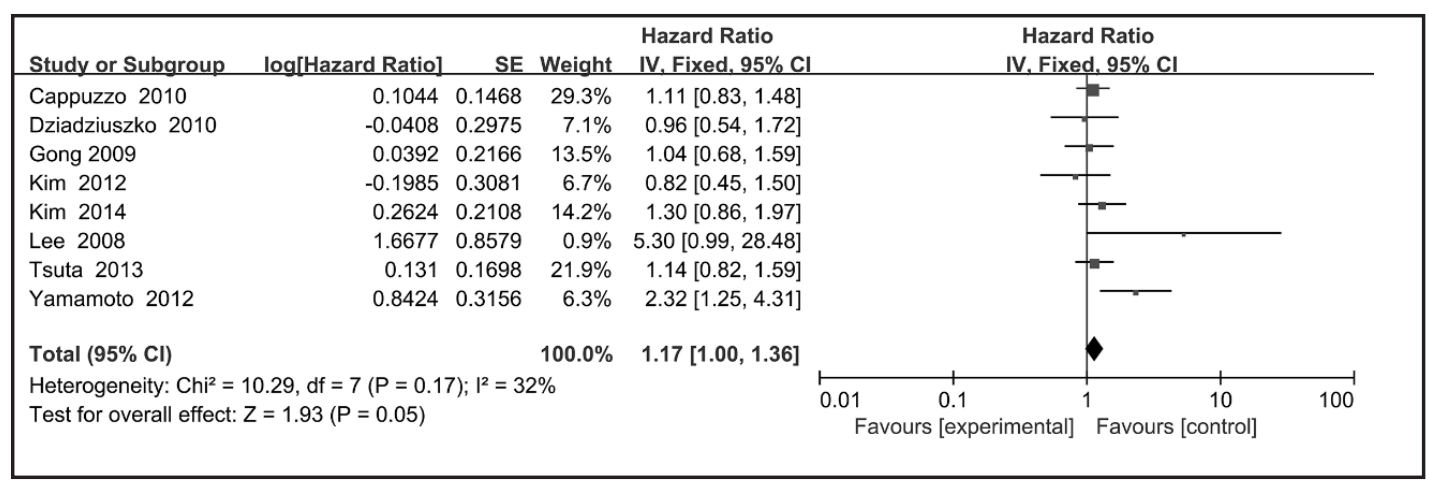

Fig. 2. Forest plot of HRs for the relationship between IGF-1R expression and OS in NSCLC. OS: overall survival, NSCLC: Non-small cell lung cancer, HR: hazard ratios.

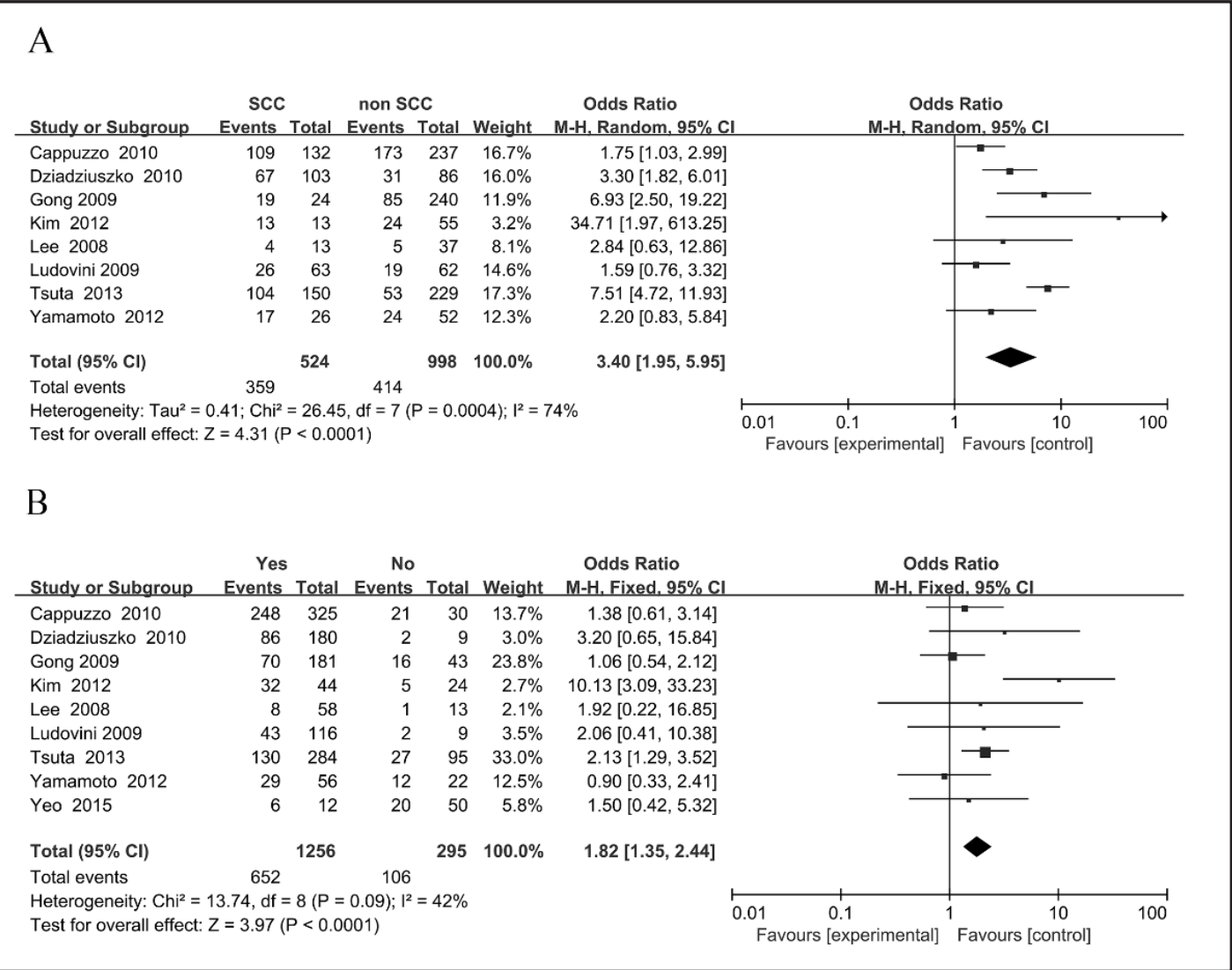

Fig. 3. Forest plot for the association between IGF-1R expression levels with clinical parameters in NSCLC. A.SCC and non-SCC; B. smoking status. SCC: squamous cell carcinoma.

62 were included. All the studies were published from 2008 to 2015 . Among these studies, 1 is from Poland [22], 1 is from USA [23], 1 is from German [24], 3 are from Japan [9, 19, 25], 2 are from Korea $[26,27]$ and 2 are from Italy $[17,28]$.

Prognostic value of IGF-1R expession in NSCLC

Eight studies investigated the relationship between IGF-1R expression and OS in total number of 1, 766 patients. Since no significant heterogeneity were exsisted among the studies ( $I^{2}=32 \%, P=0.17$ ), a fixed-effects model was used. Meta-analysis of those studies showed that high IGF-1R expression was associated with poorer OS in NSCLC (pooled HR: 1.17, 95\% CI: 1.00-1.36) (Fig. 2). 


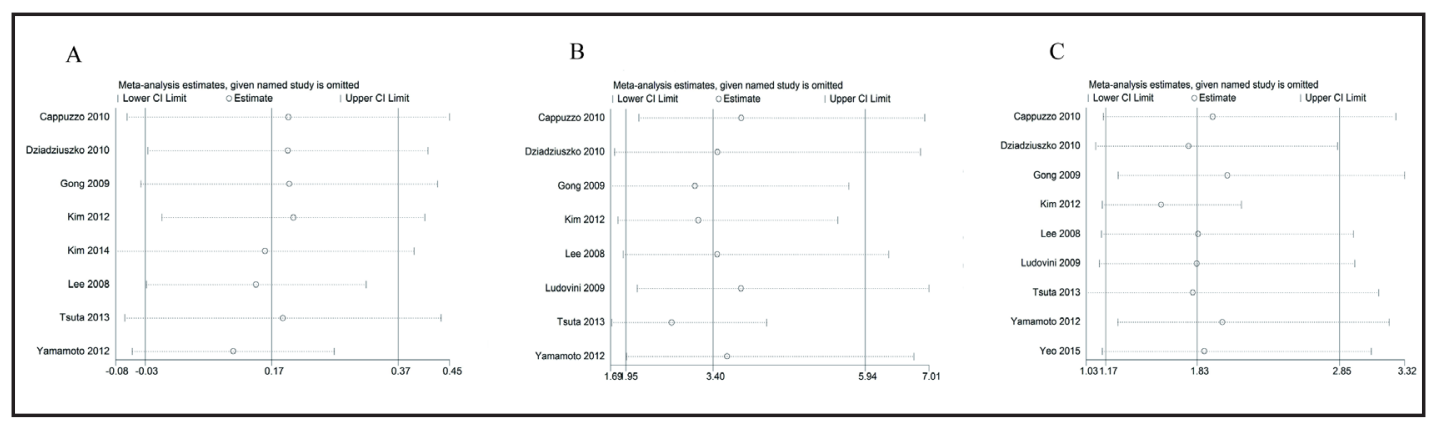

Fig. 4. Sensitivity analyses of the studies. A. overall survival; B. SCC and non-SCC; C. smoking status.

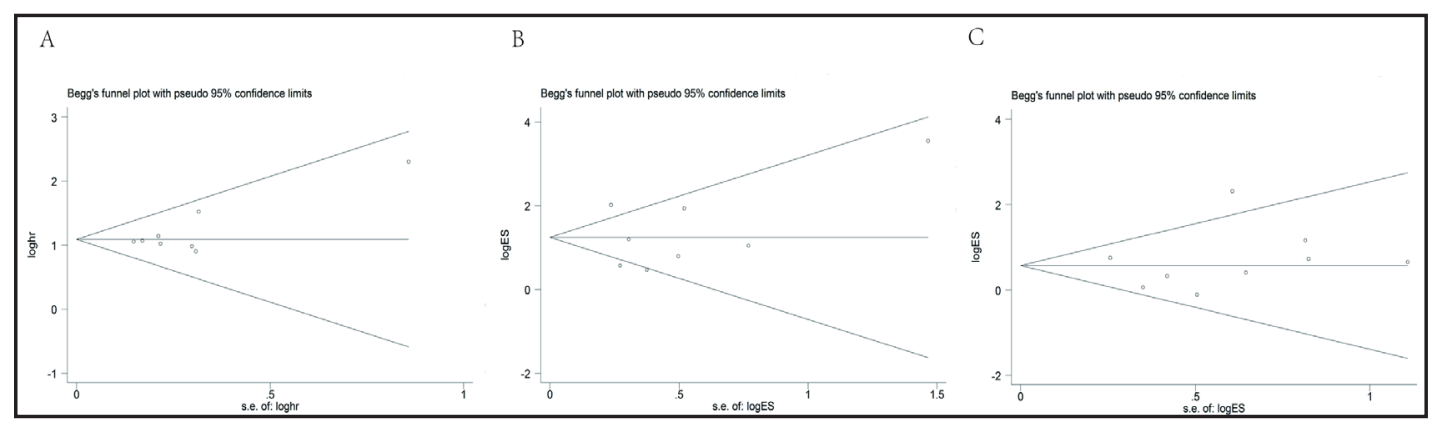

Fig. 5. Begg's test for publication bias. A. overall survival; B. SCC and non-SCC; C. smoking status.

\section{Relationship between IGF-1R and clinicopathological characteristics}

There are 8 studies investigated the expression of IGF-1R in squamous cell carcinoma (SCC) and other kind of NSCLC with a total of 1522 patients. We used random-effects model since the significant heterogeneity among studies $\left(I^{2}=74 \%, P<0.001\right)$. The pooled OR value was 3.40 (95\%CI: 1.95-5.95, Fig. 3A), which implied that IGF-1R tend to be highly expressed in squamous cell carcinoma when compared to other kinds of NSCLC.

To explore whether the expression of IGF-1R is associated with smoking status, 9 studies with a total number of 1551 patients were included. $P$ values displayed no obvious heterogeneity between the included studies $\left(I^{2}=42 \%, P=0.09\right)$. Thus, the fixed-effect model was used. Our results demonstrated that high expression of IGF-1R were significantly related to smoking status (OR=1.82, 95\% $\mathrm{CI}=1.35-2.44$, Fig. 3B).

\section{Sensitivity Analysis and Publication Bias}

Sensitivity analysis was performed to evaluate the influence of individual study on the pooled HR or ORs. One study was excluded each time and the results revealed that none of single study changed the corresponding pooled HR or ORs (Fig. 4), which conformed the reliability of our results. We used Begg's tests to evaluate the publication bias of this metaanalysis. As shown in Fig. 5, the shape of the Begg's funnel plots are symmetrical and all $P$ values $>0.05$.

\section{Discussion}

NSCLC is a common cancer worldwide. It is reported that the 5 year overall survival of NSCLC is poor and the risk of recurrence after surgical resection is high [29]. Thus identifying new biomarkers for NSCLC is urgently needed. IGF-1R is a transmembrane tyrosine kinase receptor which is activated through binding with IGF Types 1 and 2 [30]. Activated IGF$1 \mathrm{R}$ can regulate cell proliferation, differentiation, apoptosis, and transformation [31-34]. 


\section{Cellular Physiology Cell Physiol Biochem 2017;43:697-704

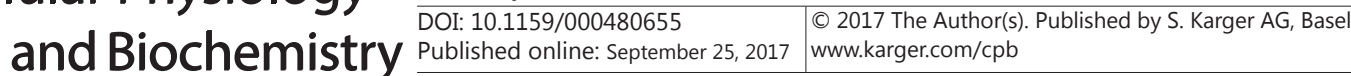 \\ Zhao et al.: Prognostic and Clinicopathological Significance of IGF-1R in NSCLC}

Many studies have reported the involvement of IGF-1R in NSCLC [19, 23, 27]. Peled et al. [35] reported that high expression of total IGF-1R can predict resistance to gefitinib with a specificity of $76 \%$ and a positive predictive value of $81 \%$ in NSCLC patients. Yamamoto et al. [9] reported that high expression of IGF-1R was associated with lymph node metastasis. All the published available articles indicated that IGF-1R played important roles in NSCLC.

A large number of studies have explored the prognostic value of IGF-1R in NSCLC, however the results were debatable. It is hard to know the exact role of IGF-1R in NSCLC, so our meta-analysis which explored the relation between IGF-1R and NSCLC is meaningful. Yamamoto et al. [9] concluded that NSCLC patients with high expression of IGF-1R tend to have poor overall survival, however, studies done by Gong [24] and Kim [19] showed no relation between IGF-1R and NSCLC overall survival, the discrepancy may be caused by the patients' different clinical stage and small sample size. By combining all the relevant articles, we got an objective and credibility conclusion that IGF-1R is associated with poor outcome in NSCLC patients. As we all know, IGF-1R can be a therapy target for cancer treatment, so we highly hold the view that it is especially better for NSCLC patients to take anti IGF-1R therapy.

There were some strengths of this study that should be noted. first, it is the first metaanalysis to analysis the relation between IGF-1R expression and clinical outcomes of NSCLC patients, second, the result of previous studies explored the association of IGF-1R and clinicopathological status of the NSCLC patients were discrepancy, by pooling all the related studies, we got a positive result, third, we found that IGF-1R was highly expressed in SCC compared to other kind of NSCLC, interestingly, a result of a randomized phase II study of IGF-1R antibody showed that the efficacy of IGF-1R antibody was prominent in SCC [18], which maybe one explanation to the above result. Despite the advantages mentioned above, there are still some limitations existed. Firstly, there were different criteria for the definition of IGF-1R positive expression, which may reduce the reliability of our results, secondly, only English language papers were included, thus the data collection may be incomplete, thirdly, only 10 studies were included in our present meta-analysis, the sample size maybe a little small, fourthly, HRs and 95\% CI were extracted from survival curves in several papers, which may generate inaccurate results. Therefore, high-quality data is urgently needed to draw more accurate conclusions.

In conclusion, our meta-analysis revealed that IGF-1R was associated with smoking status and highly expressed in SCC comparing to other kind of NSCLC, furthermore, we found that elevated expression of IGF-1R was significantly associated with poor clinical outcome. However, considering the limitations of individual study, more standardized prospective studies with high quality and large-scale are still needed to to validate our results.

\section{Disclosure Statement}

The authors declare that they have no conflicts of interest.

\section{References}

1 Torre LA, Siegel RL, Jemal A: Lung Cancer Statistics. Adv Exp Med Biol 2016;893:1-19.

2 Ferlay J, Shin HR, Bray F, Forman D, Mathers C, Parkin DM: Estimates of worldwide burden of cancer in 2008: GLOBOCAN 2008. Int J Cancer 2010;127:2893-2917.

-3 Siegel RL, Miller KD, Jemal A: Cancer Statistics, 2017. CA Cancer J Clin 2017;67:7-30.

4 Zhang R, Gu J, Chen J, Ni J, Hung J, Wang Z, Zhang X, Feng J, Ji L: High expression of PINK1 promotes proliferation and chemoresistance of NSCLC. Oncol Rep 2017;10.3892/or.2017.5486

5 Deng J, Liang Y, Liu C, He S, Wang S: The up-regulation of long non-coding RNA AFAP1-AS1 is associated with the poor prognosis of NSCLC patients. Biomed Pharmacother 2015;75:8-11.

6 Youlden DR, Cramb SM, Baade PD: The International Epidemiology of Lung Cancer: geographical distribution and secular trends. J Thorac Oncol 2008;3:819-831. 


\section{Cellular Physiology Cell Physiol Biochem 2017;43:697-704

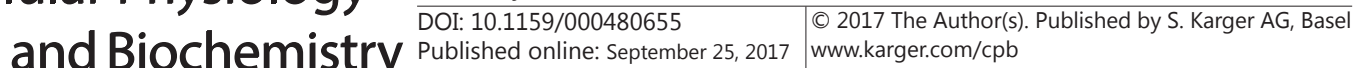

7 Khandwala HM, McCutcheon IE, Flyvbjerg A, Friend KE: The effects of insulin-like growth factors on tumorigenesis and neoplastic growth. Endocr Rev 2000;21:215-244.

8 LeRoith D, Roberts CT Jr: The insulin-like growth factor system and cancer. Cancer Lett 2003;195:127-137.

-9 Yamamoto T, Oshima T, Yoshihara K, Nishi T, Arai H, Inui K, Kaneko T, Nozawa A, Adachi H, Rino Y, Masuda M, Imada T: Clinical significance of immunohistochemical expression of insulin-like growth factor-1 receptor and matrix metalloproteinase-7 in resected non-small cell lung cancer. Exp Ther Med 2012;3:797802.

10 Wu W, Ma J, Shao N, Shi Y, Liu R, Li W, Lin Y, Wang S: Co-Targeting IGF-1R and Autophagy Enhances the Effects of Cell Growth Suppression and Apoptosis Induced by the IGF-1R Inhibitor NVP-AEW541 in TripleNegative Breast Cancer Cells. PLoS One 2017;12:e0169229.

-11 Ueda S, Tsuda H, Sato K, Takeuchi H, Shigekawa T, Matsubara O, Hiraide H, Mochizuki H: Alternative tyrosine phosphorylation of signaling kinases according to hormone receptor status in breast cancer overexpressing the insulin-like growth factor receptor type 1. Cancer Sci 2006;97:597-604.

12 Kang GH, Lee BS, Lee ES, Kim SH, Lee HY, Kang DY: Prognostic significance of p53, mTOR, c-Met, IGF-1R, and HSP70 overexpression after the resection of hepatocellular carcinoma. Gut Liver 2014;8:79-87.

13 Tang W, Feng X, Zhang S, Ren Z, Liu Y, Yang B, lv B, Cai Y, Xia J, Ge N: Caveolin-1 Confers Resistance of Hepatoma Cells to Anoikis by Activating IGF-1 Pathway. Cell Physiol Biochem 2015;36:1223-1236.

14 Nakajima N, Kozu K, Kobayashi S, Nishiyama R, Okubo R, Akai Y, Moriyama M, Kinukawa N: The expression of IGF-1R in Helicobacter pylori-infected intestinal metaplasia and gastric cancer. J Clin Biochem Nutr 2016;59:53-57.

15 Zhang X, Sun J, Wang H, Lou Y, Zhang Y, Sha H, Feng J, Han B: IGF-1R and Bmi-1 expressions in lung adenocarcinoma and their clinicopathologic and prognostic significance. Tumour Biol 2014;35:739-745.

16 Yin M, Guan X, Liao Z, Wei Q: Insulin-like growth factor-1 receptor-targeted therapy for non-small cell lung cancer: a mini review. Am J Transl Res 2009;1:101-114.

17 Cappuzzo F, Tallini G, Finocchiaro G, Wilson RS, Ligorio C, Giordano L, Toschi L, Incarbone M, Cavina R, Terracciano L, Roncalli M, Alloisio M, Varella-Garcia M, Franklin WA, Santoro A: Insulin-like growth factor receptor 1 (IGF1R) expression and survival in surgically resected non-small-cell lung cancer (NSCLC) patients. Ann Oncol 2010;21:562-567.

18 Karp DD, Paz-Ares LG, Novello S, Haluska P, Garland L, Cardenal F, Blakely LJ, Eisenberg PD, Langer CJ, Blumenschein G, Jr., Johnson FM, Green S, Gualberto A: Phase II study of the anti-insulin-like growth factor type 1 receptor antibody CP-751, 871 in combination with paclitaxel and carboplatin in previously untreated, locally advanced, or metastatic non-small-cell lung cancer. J Clin Oncol 2009;27:2516-2522.

19 Kim YH, Sumiyoshi S, Hashimoto S, Masago K, Togashi Y, Sakamori Y, Okuda C, Mio T, Mishima M: Expressions of insulin-like growth factor receptor-1 and insulin-like growth factor binding protein 3 in advanced non-small-cell lung cancer. Clin Lung Cancer 2012;13:385-390.

-20 Tierney JF, Stewart LA, Ghersi D, Burdett S, Sydes MR: Practical methods for incorporating summary timeto-event data into meta-analysis. Trials 2007;8:16.

-21 DerSimonian R, Laird N: Meta-analysis in clinical trials. Control Clin Trials 1986;7:177-188.

-22 Dziadziuszko R, Merrick DT, Witta SE, Mendoza AD, Szostakiewicz B, Szymanowska A, Rzyman W, Dziadziuszko K, Jassem J, Bunn PA, Jr., Varella-Garcia M, Hirsch FR: Insulin-like growth factor receptor 1 (IGF1R) gene copy number is associated with survival in operable non-small-cell lung cancer: a comparison between IGF1R fluorescent in situ hybridization, protein expression, and mRNA expression. J Clin Oncol 2010;28:2174-2180.

-23 Kim JS, Kim ES, Liu D, Lee JJ, Solis L, Behrens C, Lippman SM, Hong WK, Wistuba, II, Lee HY: Prognostic implications of tumoral expression of insulin like growth factors 1 and 2 in patients with non-small-cell lung cancer. Clin Lung Cancer 2014;15:213-221.

24 Gong Y, Yao E, Shen R, Goel A, Arcila M, Teruya-Feldstein J, Zakowski MF, Frankel S, Peifer M, Thomas RK, Ladanyi M, Pao W: High expression levels of total IGF-1R and sensitivity of NSCLC cells in vitro to an antiIGF-1R antibody (R1507). PLoS One 2009;4:e7273.

25 Tsuta K, Mimae T, Nitta H, Yoshida A, Maeshima AM, Asamura H, Grogan TM, Furuta K, Tsuda H: Insulinlike growth factor-1 receptor protein expression and gene copy number alterations in non-small cell lung carcinomas. Hum Pathol 2013;44:975-982. 


\section{Cellular Physiology Cell Physiol Biochem 2017;43:697-704

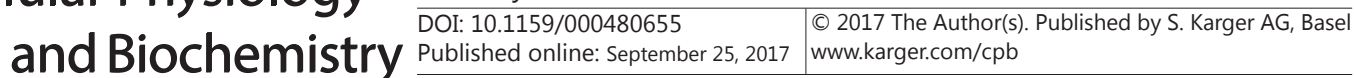

-26 Yeo CD, Park KH, Park CK, Lee SH, Kim SJ, Yoon HK, Lee YS, Lee EJ, Lee KY, Kim TJ: Expression of insulin-like growth factor 1 receptor (IGF-1R) predicts poor responses to epidermal growth factor receptor (EGFR) tyrosine kinase inhibitors in non-small cell lung cancer patients harboring activating EGFR mutations. Lung Cancer 2015;87:311-317.

-27 Lee CY, Jeon JH, Kim HJ, Shin DH, Roh TW, Ahn CM, Chang YS: Clinical significance of insulin-like growth factor-1 receptor expression in stage I non-small-cell lung cancer: immunohistochemical analysis. Korean J Intern Med 2008;23:116-120.

-28 Ludovini V, Bellezza G, Pistola L, Bianconi F, Di Carlo L, Sidoni A, Semeraro A, Del Sordo R, Tofanetti FR, Mameli MG, Daddi G, Cavaliere A, Tonato M, Crino L: High coexpression of both insulin-like growth factor receptor-1 (IGFR-1) and epidermal growth factor receptor (EGFR) is associated with shorter disease-free survival in resected non-small-cell lung cancer patients. Ann Oncol 2009;20:842-849.

29 Rami-Porta R, Crowley JJ, Goldstraw P: The revised TNM staging system for lung cancer. Ann Thorac Cardiovasc Surg 2009;15:4-9.

-30 Hubbard SR, Miller WT: Receptor tyrosine kinases: mechanisms of activation and signaling. Curr Opin Cell Biol 2007;19:117-123.

-31 Pollak M: Insulin and insulin-like growth factor signalling in neoplasia. Nat Rev Cancer 2008;8:915-928.

32 Yan S, Jiao X, Li K, Li W, Zou H: The impact of IGF-1R expression on the outcomes of patients with breast cancer: a meta-analysis. Onco Targets Ther 2015;8:279-287.

33 Lu L, Wang F, He L, Xue Y, Wang Y, Zhang H, Rong L, Wang M, Zhang Z, Fang Y, Miao H: Interaction Between IGF1 Polymorphisms and the Risk of Acute Lymphoblastic Leukemia in Chinese Children. Cell Physiol Biochem 2015;36:1346-1358.

-34 Deng Y, Wang Z, Zhang F, Qiao M, Yan Z, Wei Q, Wang J, Liu H, Fan J, Zou Y, Liao J, Hu X, Chen L, Yu X, Haydon RC, Luu HH, Qi H, He TC, Zhang J: A Blockade of IGF Signaling Sensitizes Human Ovarian Cancer Cells to the Anthelmintic Niclosamide-Induced Anti-Proliferative and Anticancer Activities. Cell Physiol Biochem 2016;39:871-888.

-35 Peled N, Wynes MW, Ikeda N, Ohira T, Yoshida K, Qian J, Ilouze M, Brenner R, Kato Y, Mascaux C, Hirsch FR: Insulin-like growth factor-1 receptor (IGF-1R) as a biomarker for resistance to the tyrosine kinase inhibitor gefitinib in non-small cell lung cancer. Cell Oncol (Dordr) 2013;36:277-288. 